\title{
Varnish Thickness Determination by Spectral Optical Coherence Tomography
}

\author{
Iwona Gorczyńska ${ }^{1}$, Maciej Wojtkowski $^{1}$, Maciej Szkulmowski ${ }^{1}$, Tomasz \\ Bajraszewski $^{1}$, Bogumiła Rouba ${ }^{2}$, Andrzej Kowalczyk $^{1}$, Piotr Targowski ${ }^{1}$ \\ ${ }^{1}$ Institute of Physics, Nicolaus Copernicus University, ul. Grudziądzka 5, 87-100 Toruń, \\ Poland \\ dranna@phys.uni.torun.pl \\ ${ }^{2}$ Institute for the Study, Restoration and Conservation of Cultural Heritage, Nicolaus \\ Copernicus University, ul. Gagarina 9, 87-100 Toruń, Poland \\ brouba@art.uni.torun.pl
}

Abstract. The applicability of Spectral Optical Coherence Tomography for non-invasive and non-contact assessment of varnish layer thickness and structure on easel paintings is discussed. The SOCT tomograms of such objects are presented.

\section{Introduction}

Knowledge of the thickness and structure of varnish layers is important for both conservation and historical reasons. It is desirable to use non-invasive and noncontact techniques for this task in order to be able to apply it as often as necessary anywhere in the picture, including the sensitive region of the artist's signature. This technique could also make possible the continuous monitoring in situ of varnish removal during laser ablation. In addition to being nondestructive, the method must be fast and simple to use, and its in-depth resolution should be better then $5 \mu \mathrm{m}$.

All the above conditions are fulfilled by Spectral domain Optical Coherence Tomography (SOCT) implemented with a broadband light source. SOCT is a rapidly developing new modality of imaging based on interference of partially coherent light: Optical Coherence Tomography (OCT). The OCT technique utilizes light sources of high spatial but low temporal coherence, such as superluminescent diodes (SLD) or femtosecond lasers. It relies on the detection of broadband light scattered from the internal structure of a semi-transparent object. The main applications of OCT remain in medicine, mainly in ophthalmology [1], but it may also be used for artworks [2-3].

\section{Experimental Methods}

The SOCT instrument is based on a fiber-optic Michelson interferometer set-up (Fig. 1). The broad-band light source LS (a Broadlighter from Superlum, Russia) is based on two coupled superluminescent diode modules with slightly shifted central wavelengths. The central wavelength of the source is $\lambda_{\text {centre }}=823 \mathrm{~nm}$ with $\Delta \lambda_{\text {FWHM }}=74 \mathrm{~nm}$. The $5 \mathrm{~mW}$ output power light source is launched into one of 
the single mode fibers of the 50:50 fiber coupler DC through an optical isolator OI. The optical isolator separates the light source from the light back-reflected from the elements of the interferometer. The light is split by the coupler into two arms: the reference and object. The former is equipped with a polarization controller PC, collimator and open air delay line with a reflective mirror RM kept in a fixed position. The latter (an object head) consists of a collimator, transversal scanners $X-Y$ and lenses $L$ and $L_{1}$. The lens $L_{1}$ is placed between the scanner and an object so the distances, lens-to-object and from pivot point of the scanner to the lens, are equal to the focal length of the lens.

The optics produces a narrow beam of light of high spatial but low temporal coherence. This beam penetrates the object, scatters from elements of its structure, then is collected by the same optics $\mathrm{L}$ and $\mathrm{L}_{1}$ back to the coupler DC. Then it interferes with the light returning from the reference arm and the interference signal is directed into a custom-designed spectrometer. It consists of a volume phase holographic grating DG with 1200 lines/mm and achromatic lens $\mathrm{L}(\mathrm{f}=150 \mathrm{~mm})$ which focuses the spectrum on a 12-bit line scan CCD camera. The spectral fringe patterns registered by this detector are then transferred to a personal computer COMP. The resulting signal, i.e. spectral fringe patterns, is Fourier-transformed into a single line of a cross-sectional image (A-scan). In order to obtain either 2D slice (B-scan, Fig. 2a, Fig. 3) or 3D volume tomogram, the beam is scanned transversally by galvanometric scanners $\mathrm{X}-\mathrm{Y}$.

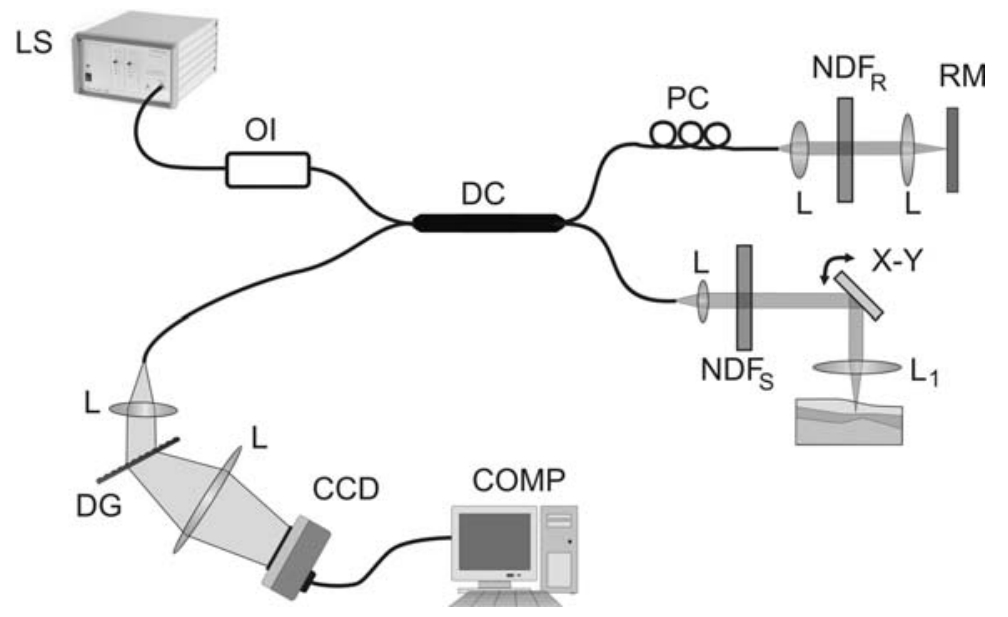

Fig. 1. The set-up for a Spectral OCT instrument. LS: light source, OI: optical isolator, DC: directional coupler, PC: polarization controller, NDF: neutral density filters, RM: reference mirror, X-Y: scanners, L: lenses, DG: diffraction grating, CCD: linear CCD camera

The system operates in the shot-noise-limited detection mode (the intensity of light in the reference arm of interferometer is controlled by neutral density filter NDF) with sensitivity of $90 \mathrm{~dB}$. The exposure time per A-scan is equal to $50 \mu \mathrm{s}$, thus one 2D slice (composed usually of 2000 to 5000 A-scans) is collected in a 
fraction of second. The numerical processing of the data, besides the Fourier transformation essential to the SOCT method, includes subtraction of noninterference background, spectral shaping [4], and numerical dispersion correction [5]. The signal may be visualized as a cross sectional view (B-scan), or converted into a variety of 3D images, like a map of varnish thickness (Fig. 4).

In Optical Coherence Tomography generally, the axial resolution $\Delta \mathrm{z}$ is decoupled from the lateral resolution $\Delta x$. The first depends on the properties of the light source:

$$
\Delta z=\frac{1}{n_{R}} \frac{2 \ln 2}{\pi} \frac{\lambda_{\text {centre }}^{2}}{\Delta \lambda_{\text {FWHM }}}
$$

while the second is determined by the properties of the optical head:

$$
\Delta x=\frac{1}{n_{R}} \frac{4 \lambda_{\text {centre }}}{\pi}\left(\frac{f}{d}\right),
$$

where $n_{R}$ is the refractive index of the examined media, $f$ is the focal length of the lens $\mathrm{L}_{1}$, and $d$ is the beam diameter at this lens. For the system described here: $\Delta \mathrm{z}$ $=6 \mu \mathrm{m}$ in a layer of varnish and $\Delta \mathrm{x}=15 \mu \mathrm{m}$. The power of infrared light illuminating the sample is about $600 \mu \mathrm{W}$.

The object chosen for examination was an $18^{\text {th }}$ century painting on wood panel (The Netherlands school). A sample for microscopic analysis of cross-section has been taken from the picture in an area of destruction.

\section{Results and Discussion}

In Fig. 2, an example of a 2D slice obtained with SOCT is presented and compared with microscopic image of the cross-section of a sample taken nearby.
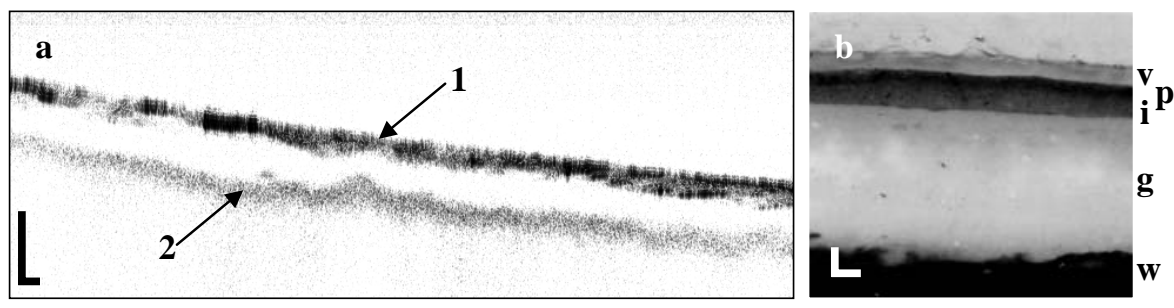

Fig. 2. (a) The SOCT image of a cross-section of painting layer covered by varnish. The dark bands correspond to strong light scattering at (1) the air-varnish and (2) varnish-paint layer interfaces. The light penetrates from the top, the structures below the varnish layer are not visible due to high absorption of paint. (b) The microscopic image of the cross-section of a sample taken from the same object. The following layers are visible: varnish (v), paint layer (p), imprimatura (i), ground (g), wood (w). The bar scales are $100 \mu \mathrm{m}$ in both directions in both images 
The direct microscopic analysis of cross-section (Fig. 2b) obtained from the same picture indicates the presence of about $70 \mu \mathrm{m}$ thick varnish layer. The same thickness is obtained from OCT measurement. The chemical assay indicated the oil-and-resin varnish, not tinted.

In Fig. 3, a different area of the same picture is analysed. Here, a strong impasto of a white paint is elevated over the varnish surface. With OCT imaging it is possible to precisely access this detail of picture structure.

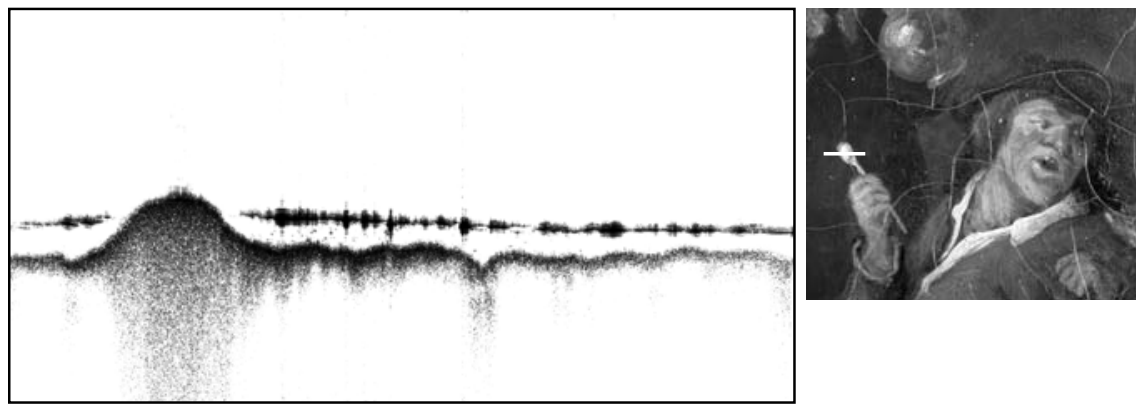

Fig. 3. The SOCT cross-section image taken across a white-painted pipe

It is worthwhile to emphasise, that all in-depth distances at OCT tomograms are optical ones. To adjust for this effect, a refraction correction [6] is sometimes necessary. In Fig. 4, an example of this effect is presented.
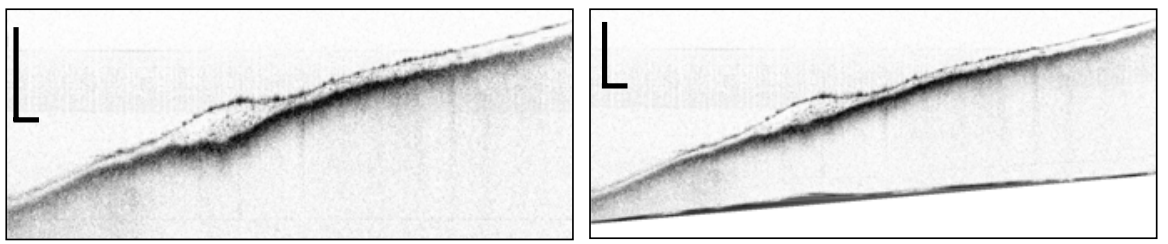

Fig. 4. The effect of refraction correction. (Left) an uncorrected image, bars indicate $0.2 \mathrm{~mm}$ in varnish $(\mathrm{n}=1.5)$. (Right) a corrected image, bars indicate $0.2 \mathrm{~mm}$ of geometrical distances. The picture examined was tilted to eliminate specular reflection from the varnish layer

If the surface is scanned in both $(\mathrm{X}, \mathrm{Y})$ directions, the information on volume structure of the object becomes available. From every 2D slice, both interfaces (marked 1 and 2 in Fig. 2a) have been automatically recognised and then linked together into two surface maps. The lower one represents a painting layer surface under the varnish while the upper one demonstrates a final picture surface. A point-to-point map of distances between both interfaces shows the varnish layer thickness.

To prepare images presented in Fig 5, a set of 54 subsequent, parallel slices (B-scans), each composed of 2000 A-scans, was collected. Then both interfaces were recovered and varnish thickness map calculated. In Fig. 5a, for clearer presentation, only the paint layer surface is shown. 

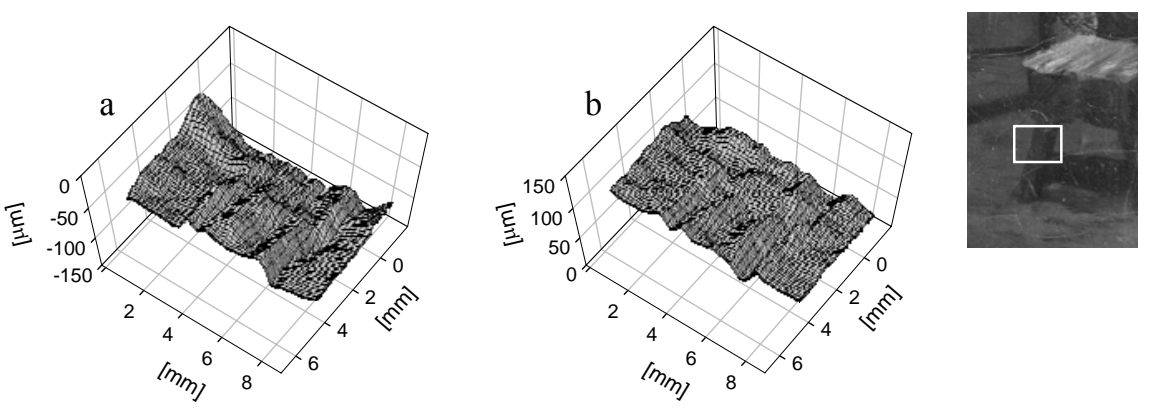

Fig. 5. The surface map of a painting layer (a) under the varnish and (b) a varnish thickness map as revealed by SOCT examination

\section{Conclusions}

The study presented confirms that, under defined technical conditions, SOCT can be used for determination of varnish layer thickness. The major limitation is the axial resolution of the method, demanding use of expensive, very broad, but spatially coherent light sources. However, progress in developing multi-SLD sources promises rapid reduction of their price. Then, in the future, this technique may be established as a routine method in art examination, as it is at present in medicine. Since the method is non-destructive, non-invasive and instantaneous, it may be used in particular in combination with laser ablation devices for the online control of varnish removal processes.

Acknowledgement. This work was supported by Polish Ministry of Science with Grant 2 H01E 02525.

\section{References}

1 J.S. Schuman, C.A. Puliafito and J.G. Fujimoto, Optical Coherence Tomography of Ocular Diseases, 2nd ed., Slack Inc, Thorofare, NJ, USA, 2004

2 P. Targowski, B. Rouba, M. Wojtkowski, A. Kowalczyk, Studies in Conservation, 49, 107-114, 2004

3 H. Liang, M. G. Cid, R. G. Cucu, G. M. Dobre, A. G. Podoleanu, J. Pedro, and D. Saunders, Opt. Express 13, 6133-6144, 2005

4 M. Szkulmowski, M. Wojtkowski, P.Targowski, A. Kowalczyk, Proc.SPIE 5316, 424-431, 2004

5 B. Cense, N.A. Nassif, T. C. Chen, M.C.Pierce, S-H. Yun, B.H. Park,B. E.Bouma, G.J. Tearney, J.F. de Boer, Opt. Express 12, 2435-2447, 2004

6 A. Szkulmowska, M. Góra, M. Targowska, B. Rouba, D. Stifter, E. Breuer, P. Targowski, in this volume 